Acta Crystallographica Section E

Structure Reports

Online

ISSN 1600-5368

\section{2-(2-Fluoro-4-nitrophenoxy)-3-nitro- pyridine}

\section{Lili Cui* and Xingquan He}

Department of Chemistry and Chemical Engineering, Changchun University of Science and Technology, Changchun 130022, People's Republic of China

Correspondence e-mail: cuilili1127@gmail.com

Received 11 November 2013; accepted 19 December 2013

Key indicators: single-crystal X-ray study; $T=293 \mathrm{~K}$; mean $\sigma(\mathrm{C}-\mathrm{C})=0.004 \AA$; $R$ factor $=0.062 ; w R$ factor $=0.140 ;$ data-to-parameter ratio $=14.4$.

In the title compound, $\mathrm{C}_{11} \mathrm{H}_{6} \mathrm{FN}_{3} \mathrm{O}_{5}$, the dihedral angle between the aromatic rings is $72.4(3)^{\circ}$. The $\mathrm{NO}_{2}$ groups form dihedral angles of 40.8 (2) and $4.8(2)^{\circ}$, respectively, with the attached pyridine and benzene rings. The crystal structure features $\pi-\pi$ stacking between centrosymmetrically related pairs of pyridine rings [centroid-centroid separation = 3.800 (3) Å].

\section{Related literature}

For applications and the biological activity of 2-phenoxypyridine, see: Chao et al. (2013).<smiles>O=[N+]([O-])c1ccc(Oc2ncccc2[N+](=O)[O-])c(F)c1</smiles>

\section{Experimental}

\section{Crystal data}

$\mathrm{C}_{11} \mathrm{H}_{6} \mathrm{FN}_{3} \mathrm{O}_{5} \quad M_{r}=279.19$
Monoclinic, $P 2_{1} / c$
$a=7.5275(15) \AA$
$b=21.804(4) \AA$
$c=7.1681(14) \AA$
$\beta=101.07(3)^{\circ}$
$V=1154.6(4) \AA^{3}$

\section{Data collection}

Rigaku R-AXIS RAPID IP diffractometer

Absorption correction: multi-scan $T_{\min }=0.945, T_{\max }=0.970$

Refinement

$R\left[F^{2}>2 \sigma\left(F^{2}\right)\right]=0.062$

$w R\left(F^{2}\right)=0.140$

$S=0.94$

2611 reflections (ABSCOR; Higashi, 1995)
$Z=4$

Mo $K \alpha$ radiation

$\mu=0.14 \mathrm{~mm}^{-1}$

$T=293 \mathrm{~K}$

$0.41 \times 0.36 \times 0.22 \mathrm{~mm}$

10606 measured reflections 2611 independent reflections 1351 reflections with $I>2 \sigma(I)$ $R_{\text {int }}=0.083$

181 parameters

$\mathrm{H}$-atom parameters constrained

$\Delta \rho_{\max }=0.21$ e $\AA^{-3}$

$\Delta \rho_{\min }=-0.26 \mathrm{e}^{-3}$
Data collection: RAPID-AUTO (Rigaku, 1998); cell refinement: RAPID-AUTO; data reduction: RAPID-AUTO; program(s) used to solve structure: SHELXS97 (Sheldrick, 2008); program(s) used to refine structure: SHELXL97 (Sheldrick, 2008); molecular graphics: SHELXTL (Sheldrick, 2008); software used to prepare material for publication: SHELXTL.

The project was sponsored by the Natural Science Foundation of China (No. 21273024) and the Natural Science Foundation of Jilin Province, China (No. 201215135).

Supplementary data and figures for this paper are available from the IUCr electronic archives (Reference: PK2504).

\section{References}

Chao, H., Turdi, H., Herpin, T. F., Roberge, J. Y., Liu, Y., Schnur, D. M., Poss, M. A., Rehfuss, R., Hua, J., Wu, Q., Price, L. A., Abell, L. M., Schumacher, W. A., Bostwick, J. S., Steinbacher, T. E., Stewart, A. B., Ogletree, M. L., Huang, C. S., Chang, M., Cacace, A. M., Arcuri, M. J., Celani, D., Wexler, R. R. \& Lawrence, R. M. (2013). J. Med. Chem. 56, 1704-1714. Higashi, T. (1995). ABSCOR. Rigaku Corporation, Tokyo, Japan. Rigaku (1998). RAPID-AUTO. Rigaku Corporation, Tokyo, Japan. Sheldrick, G. M. (2008). Acta Cryst. A64, 112-122. 


\section{supporting information}

Acta Cryst. (2014). E70, o100 [https://doi.org/10.1107/S1600536813034181]

\section{2-(2-Fluoro-4-nitrophenoxy)-3-nitropyridine}

\section{Lili Cui and Xingquan He}

\section{S1. Comment}

2-Phenoxypyridines have been shown to be small molecule P2Y1 antagonists (Chao et al. 2013). Here, the crystal structure of 2-(2-fluoro-4-nitrophenoxy)- 3-nitropyridine is reported.

\section{S2. Experimental}

To a solution of 2-chloro-3-nitropyridine (1.0 g, $6.3 \mathrm{mmol})$ and 2-fluoro-4-nitrophenol (1.48 g, $9.45 \mathrm{mmol})$ in toluene (35 $\mathrm{ml}$ ) was added $N, N$-diisopropylethylamine $(3.3 \mathrm{ml}, 18.9 \mathrm{mmol})$. The reaction mixture was stirred for $48 \mathrm{~h}$ under reflux. The reaction mixture was concentrated in vacuo, diluted with water, and extracted with EtOAc. The organic phase was washed with brine, dried over anhydrous $\mathrm{MgSO}_{4}$, and concentrated in vacuo to yield the crude product as a solid. Purification by recrystallization from methanol gave the desired product, 2-(2-fluoro-4-nitrophenoxy)-3- nitropyridine (yellow solid, $0.80 \mathrm{~g}, 46 \%, 97.1-98.4{ }^{\circ} \mathrm{C}$ ). ${ }^{1} \mathrm{H}$ NMR (DMSO- $d 6,300 \mathrm{~Hz}$ ), 8.70 (dd, J = 8.1, 1.8 Hz, 1H), 8.47 (dd, J = 4.8, $1.8 \mathrm{~Hz}, 1 \mathrm{H}), 8.41(\mathrm{dd}, \mathrm{J}=10.2,2.7 \mathrm{~Hz}, 1 \mathrm{H}), 8.25-8.20(\mathrm{~m}, 1 \mathrm{H}), 7.80-7.74(\mathrm{~m} \mathrm{1H}), 7.54-7.50(\mathrm{~m}, 1 \mathrm{H})$; ES-MS: 279.8 $\left[\left(M+\mathrm{H}^{+}\right)\right]$. Crystals of the title compound for X-ray diffraction were obtained by slow evaporation of $\mathrm{MeOH} / \mathrm{CH}_{2} \mathrm{Cl}_{2}$ solution.

\section{S3. Refinement}

All hydrogen atoms were fixed at calculated positions and refined by using a riding model with $\mathrm{C}-\mathrm{H}=0.93 \AA$ and $U_{\text {iso }}(\mathrm{H})=1.2 U_{\text {eq }}(\mathrm{C})$. 


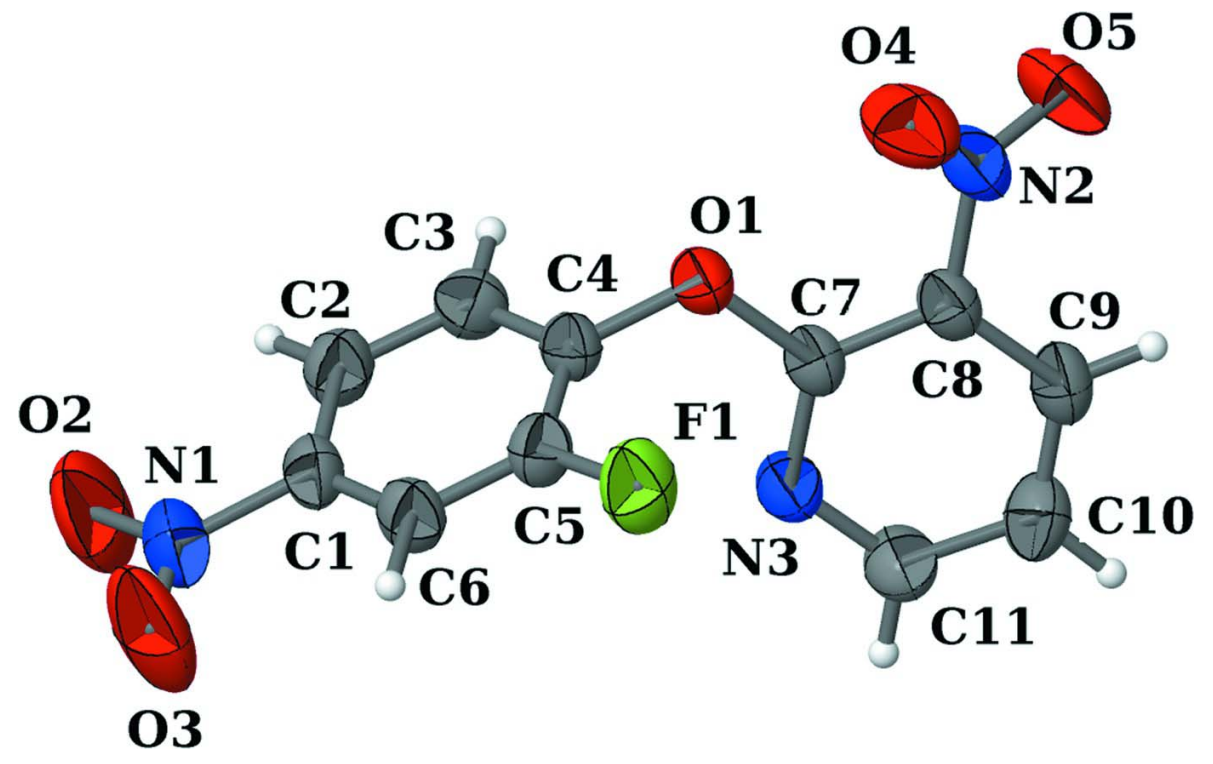

Figure 1

The molecular structure of the title compound. Displacement ellipsoids are shown at the $50 \%$ probability level.

\section{2-(2-Fluoro-4-nitrophenoxy)-3-nitropyridine}

Crystal data

$\mathrm{C}_{11} \mathrm{H}_{6} \mathrm{FN}_{3} \mathrm{O}_{5}$

$M_{r}=279.19$

Monoclinic, $P 2_{1} / c$

Hall symbol: -P $2 \mathrm{ybc}$

$a=7.5275(15) \AA$

$b=21.804(4) \AA$

$c=7.1681$ (14) $\AA$

$\beta=101.07(3)^{\circ}$

$V=1154.6(4) \AA^{3}$

$Z=4$

Data collection

Rigaku R-AXIS RAPID IP diffractometer

Radiation source: fine-focus sealed tube

Graphite monochromator

Detector resolution: 0.1 pixels $\mathrm{mm}^{-1}$

oscillation scans

Absorption correction: multi-scan

(ABSCOR; Higashi, 1995)

$T_{\min }=0.945, T_{\max }=0.970$

\section{Refinement}

Refinement on $F^{2}$

Least-squares matrix: full

$R\left[F^{2}>2 \sigma\left(F^{2}\right)\right]=0.062$

$w R\left(F^{2}\right)=0.140$

$S=0.94$

2611 reflections

181 parameters
$F(000)=568$

$D_{\mathrm{x}}=1.606 \mathrm{Mg} \mathrm{m}^{-3}$

Melting point $=370.1-371.4 \mathrm{~K}$

Mo $K \alpha$ radiation, $\lambda=0.71073 \AA$

Cell parameters from 7455 reflections

$\theta=3.1-27.7^{\circ}$

$\mu=0.14 \mathrm{~mm}^{-1}$

$T=293 \mathrm{~K}$

Block, colourless

$0.41 \times 0.36 \times 0.22 \mathrm{~mm}$

10606 measured reflections

2611 independent reflections

1351 reflections with $I>2 \sigma(I)$

$R_{\text {int }}=0.083$

$\theta_{\max }=27.5^{\circ}, \theta_{\min }=3.0^{\circ}$

$h=-9 \rightarrow 9$

$k=-28 \rightarrow 28$

$l=-9 \rightarrow 8$

0 restraints

Primary atom site location: structure-invariant direct methods

Secondary atom site location: difference Fourier map

Hydrogen site location: inferred from neighbouring sites 
H-atom parameters constrained $w=1 /\left[\sigma^{2}\left(F_{\mathrm{o}}^{2}\right)+(0.0498 P)^{2}\right]$

where $P=\left(F_{\mathrm{o}}^{2}+2 F_{\mathrm{c}}{ }^{2}\right) / 3$

$$
\begin{aligned}
& (\Delta / \sigma)_{\max }<0.001 \\
& \Delta \rho_{\max }=0.21 \mathrm{e} \AA^{-3} \\
& \Delta \rho_{\min }=-0.26 \mathrm{e} \AA^{-3}
\end{aligned}
$$

Special details

Geometry. All e.s.d.'s (except the e.s.d. in the dihedral angle between two l.s. planes) are estimated using the full covariance matrix. The cell e.s.d.'s are taken into account individually in the estimation of e.s.d.'s in distances, angles and torsion angles; correlations between e.s.d.'s in cell parameters are only used when they are defined by crystal symmetry. An approximate (isotropic) treatment of cell e.s.d.'s is used for estimating e.s.d.'s involving 1.s. planes.

Refinement. Refinement of $F^{2}$ against ALL reflections. The weighted $R$-factor $w R$ and goodness of fit $S$ are based on $F^{2}$, conventional $R$-factors $R$ are based on $F$, with $F$ set to zero for negative $F^{2}$. The threshold expression of $F^{2}>\sigma\left(F^{2}\right)$ is used only for calculating $R$-factors $(\mathrm{gt})$ etc. and is not relevant to the choice of reflections for refinement. $R$-factors based on $F^{2}$

\begin{tabular}{|c|c|c|c|c|}
\hline & $x$ & $y$ & $z$ & $U_{\text {iso }} * / U_{\text {eq }}$ \\
\hline $\mathrm{C} 5$ & $0.7119(3)$ & $0.37835(11)$ & $0.5574(3)$ & $0.0470(6)$ \\
\hline $\mathrm{C} 4$ & $0.8649(3)$ & $0.34302(12)$ & $0.6136(3)$ & $0.0453(6)$ \\
\hline $\mathrm{C} 1$ & $0.5475(3)$ & $0.29000(12)$ & $0.4494(3)$ & $0.0492(6)$ \\
\hline $\mathrm{C} 3$ & $0.8592(3)$ & $0.28155(12)$ & $0.5825(3)$ & $0.0535(7)$ \\
\hline H3 & 0.9634 & 0.2580 & 0.6169 & $0.064^{*}$ \\
\hline C6 & $0.5501(3)$ & $0.35242(12)$ & $0.4752(3)$ & $0.0516(7)$ \\
\hline H6 & 0.4464 & 0.3761 & 0.4385 & $0.062 *$ \\
\hline $\mathrm{C} 2$ & $0.6968(3)$ & $0.25381(13)$ & $0.4988(4)$ & $0.0554(7)$ \\
\hline $\mathrm{H} 2$ & 0.6904 & 0.2117 & 0.4772 & $0.066^{*}$ \\
\hline $\mathrm{C} 7$ & $1.0406(3)$ & $0.39397(11)$ & $0.8728(3)$ & $0.0430(6)$ \\
\hline $\mathrm{C} 8$ & $1.1950(3)$ & $0.42593(11)$ & $0.9561(3)$ & $0.0446(6)$ \\
\hline C9 & $1.2093(3)$ & $0.44800(12)$ & $1.1372(3)$ & $0.0524(6)$ \\
\hline H9 & 1.3124 & 0.4691 & 1.1956 & $0.063^{*}$ \\
\hline $\mathrm{C} 10$ & $1.0688(4)$ & $0.43849(13)$ & $1.2313(3)$ & $0.0598(7)$ \\
\hline $\mathrm{H} 10$ & 1.0732 & 0.4535 & 1.3536 & $0.072 *$ \\
\hline C11 & $0.9221(4)$ & $0.40613(13)$ & $1.1387(3)$ & $0.0580(7)$ \\
\hline H11 & 0.8268 & 0.3995 & 1.2018 & $0.070 *$ \\
\hline $\mathrm{F} 1$ & $0.7237(2)$ & $0.43888(7)$ & $0.5831(2)$ & 0.0720 \\
\hline $\mathrm{O} 1$ & $1.0278(2)$ & $0.37145(9)$ & $0.6923(2)$ & $0.0550(5)$ \\
\hline $\mathrm{O} 4$ & $1.3069(3)$ & $0.45113(12)$ & $0.6873(3)$ & $0.0846(7)$ \\
\hline $\mathrm{O} 5$ & $1.4970(2)$ & $0.43310(12)$ & $0.9461(4)$ & $0.0937(8)$ \\
\hline $\mathrm{O} 2$ & $0.3673(3)$ & $0.20648(13)$ & $0.3537(5)$ & $0.1251(11)$ \\
\hline $\mathrm{O} 3$ & $0.2448(3)$ & $0.29424(13)$ & $0.3109(4)$ & $0.1255(12)$ \\
\hline N3 & $0.9075(3)$ & $0.38343(10)$ & $0.9628(3)$ & $0.0508(5)$ \\
\hline N2 & $1.3435(3)$ & $0.43708(10)$ & $0.8543(4)$ & $0.0593(6)$ \\
\hline N1 & $0.3735(3)$ & $0.26136(14)$ & $0.3662(4)$ & $0.0716(7)$ \\
\hline
\end{tabular}
are statistically about twice as large as those based on $F$, and $R$-factors based on ALL data will be even larger.

Fractional atomic coordinates and isotropic or equivalent isotropic displacement parameters $\left(\AA^{2}\right)$

Atomic displacement parameters $\left(\AA^{2}\right)$

\begin{tabular}{lllllll}
\hline & $U^{11}$ & $U^{22}$ & $U^{33}$ & $U^{12}$ & $U^{13}$ & $U^{23}$ \\
\hline C5 & $0.0507(13)$ & $0.0405(14)$ & $0.0488(12)$ & $-0.0032(11)$ & $0.0074(11)$ & $-0.0045(10)$ \\
C4 & $0.0391(12)$ & $0.0565(16)$ & $0.0385(11)$ & $-0.0039(11)$ & $0.0029(10)$ & $-0.0049(10)$
\end{tabular}




$\begin{array}{lllllll}\text { C1 } & 0.0444(13) & 0.0523(16) & 0.0473(13) & -0.0054(11) & -0.0005(11) & -0.0089(11) \\ \text { C3 } & 0.0452(13) & 0.0559(17) & 0.0568(14) & 0.0103(11) & 0.0034(12) & -0.0036(12) \\ \text { C6 } & 0.0428(13) & 0.0531(17) & 0.0541(14) & 0.0053(11) & -0.0028(11) & -0.0027(11) \\ \text { C2 } & 0.0579(15) & 0.0461(16) & 0.0600(15) & 0.0013(12) & 0.0060(13) & -0.0083(11) \\ \text { C7 } & 0.0374(12) & 0.0461(14) & 0.0429(11) & -0.0031(10) & 0.0009(10) & -0.0003(10) \\ \text { C8 } & 0.0356(11) & 0.0426(14) & 0.0526(13) & -0.0010(10) & 0.0008(11) & 0.0020(10) \\ \text { C9 } & 0.0477(13) & 0.0484(15) & 0.0546(14) & -0.0047(11) & -0.0060(12) & -0.0031(11) \\ \text { C10 } & 0.0678(17) & 0.0625(18) & 0.0448(13) & -0.0060(14) & 0.0003(13) & -0.0058(12) \\ \text { C11 } & 0.0575(15) & 0.0730(19) & 0.0447(13) & -0.0122(14) & 0.0126(12) & -0.0008(12) \\ \text { F1 } & 0.0736(10) & 0.0425(9) & 0.0955(11) & -0.0038(7) & 0.0051(9) & -0.0074(8) \\ \text { O1 } & 0.0392(9) & 0.0785(13) & 0.0472(9) & -0.0119(8) & 0.0080(7) & -0.0158(8) \\ \text { O4 } & 0.0682(13) & 0.116(2) & 0.0747(13) & -0.0145(13) & 0.0261(11) & 0.0135(13) \\ \text { O5 } & 0.0355(10) & 0.121(2) & 0.1207(18) & -0.0093(11) & 0.0043(11) & -0.0072(15) \\ \text { O2 } & 0.0874(18) & 0.0756(19) & 0.202(3) & -0.0328(14) & 0.0027(18) & -0.0372(19) \\ \text { O3 } & 0.0584(14) & 0.109(2) & 0.183(3) & -0.0043(14) & -0.0421(17) & -0.0136(19) \\ \text { N3 } & 0.0449(11) & 0.0617(14) & 0.0457(10) & -0.0105(10) & 0.0086(9) & -0.0026(9) \\ \text { N2 } & 0.0392(11) & 0.0564(15) & 0.0805(16) & -0.0078(10) & 0.0073(11) & -0.0048(12) \\ \text { N1 } & 0.0568(15) & 0.0735(19) & 0.0797(16) & -0.0166(13) & 0.0011(13) & -0.0196(14) \\ & & & & & & \end{array}$

Geometric parameters $\left(\AA,{ }^{\circ}\right)$

\begin{tabular}{|c|c|c|c|}
\hline $\mathrm{C} 5-\mathrm{F} 1$ & $1.333(3)$ & $\mathrm{C} 7-\mathrm{C} 8$ & $1.388(3)$ \\
\hline $\mathrm{C} 5-\mathrm{C} 6$ & $1.369(3)$ & $\mathrm{C} 8-\mathrm{C} 9$ & $1.369(3)$ \\
\hline $\mathrm{C} 5-\mathrm{C} 4$ & $1.380(3)$ & $\mathrm{C} 8-\mathrm{N} 2$ & $1.468(3)$ \\
\hline $\mathrm{C} 4-\mathrm{C} 3$ & $1.358(3)$ & $\mathrm{C} 9-\mathrm{C} 10$ & $1.375(4)$ \\
\hline $\mathrm{C} 4-\mathrm{O} 1$ & $1.393(3)$ & C9- $\mathrm{H} 9$ & 0.9300 \\
\hline $\mathrm{C} 1-\mathrm{C} 2$ & $1.363(3)$ & $\mathrm{C} 10-\mathrm{C} 11$ & $1.370(3)$ \\
\hline $\mathrm{C} 1-\mathrm{C} 6$ & $1.373(3)$ & $\mathrm{C} 10-\mathrm{H} 10$ & 0.9300 \\
\hline $\mathrm{C} 1-\mathrm{N} 1$ & $1.470(3)$ & $\mathrm{C} 11-\mathrm{N} 3$ & $1.339(3)$ \\
\hline $\mathrm{C} 3-\mathrm{C} 2$ & $1.392(3)$ & $\mathrm{C} 11-\mathrm{H} 11$ & 0.9300 \\
\hline $\mathrm{C} 3-\mathrm{H} 3$ & 0.9300 & $\mathrm{O} 4-\mathrm{N} 2$ & $1.215(3)$ \\
\hline $\mathrm{C} 6-\mathrm{H} 6$ & 0.9300 & $\mathrm{O} 5-\mathrm{N} 2$ & $1.218(2)$ \\
\hline $\mathrm{C} 2-\mathrm{H} 2$ & 0.9300 & $\mathrm{O} 2-\mathrm{N} 1$ & $1.200(3)$ \\
\hline $\mathrm{C} 7-\mathrm{N} 3$ & $1.312(3)$ & $\mathrm{O} 3-\mathrm{N} 1$ & $1.209(3)$ \\
\hline $\mathrm{C} 7-\mathrm{O} 1$ & $1.370(3)$ & & \\
\hline $\mathrm{F} 1-\mathrm{C} 5-\mathrm{C} 6$ & $119.9(2)$ & $\mathrm{C} 9-\mathrm{C} 8-\mathrm{C} 7$ & $119.4(2)$ \\
\hline $\mathrm{F} 1-\mathrm{C} 5-\mathrm{C} 4$ & $118.9(2)$ & $\mathrm{C} 9-\mathrm{C} 8-\mathrm{N} 2$ & $118.9(2)$ \\
\hline $\mathrm{C} 6-\mathrm{C} 5-\mathrm{C} 4$ & $121.3(2)$ & $\mathrm{C} 7-\mathrm{C} 8-\mathrm{N} 2$ & $121.6(2)$ \\
\hline $\mathrm{C} 3-\mathrm{C} 4-\mathrm{C} 5$ & $120.3(2)$ & $\mathrm{C} 8-\mathrm{C} 9-\mathrm{C} 10$ & $119.0(2)$ \\
\hline $\mathrm{C} 3-\mathrm{C} 4-\mathrm{O} 1$ & $120.3(2)$ & $\mathrm{C} 8-\mathrm{C} 9-\mathrm{H} 9$ & 120.5 \\
\hline $\mathrm{C} 5-\mathrm{C} 4-\mathrm{O} 1$ & $119.3(2)$ & $\mathrm{C} 10-\mathrm{C} 9-\mathrm{H} 9$ & 120.5 \\
\hline $\mathrm{C} 2-\mathrm{C} 1-\mathrm{C} 6$ & $123.3(2)$ & $\mathrm{C} 11-\mathrm{C} 10-\mathrm{C} 9$ & $117.6(2)$ \\
\hline $\mathrm{C} 2-\mathrm{C} 1-\mathrm{N} 1$ & $119.0(2)$ & $\mathrm{C} 11-\mathrm{C} 10-\mathrm{H} 10$ & 121.2 \\
\hline $\mathrm{C} 6-\mathrm{C} 1-\mathrm{N} 1$ & $117.7(2)$ & $\mathrm{C} 9-\mathrm{C} 10-\mathrm{H} 10$ & 121.2 \\
\hline $\mathrm{C} 4-\mathrm{C} 3-\mathrm{C} 2$ & $119.8(2)$ & $\mathrm{N} 3-\mathrm{C} 11-\mathrm{C} 10$ & $124.0(3)$ \\
\hline $\mathrm{C} 4-\mathrm{C} 3-\mathrm{H} 3$ & 120.1 & $\mathrm{~N} 3-\mathrm{C} 11-\mathrm{H} 11$ & 118.0 \\
\hline $\mathrm{C} 2-\mathrm{C} 3-\mathrm{H} 3$ & 120.1 & $\mathrm{C} 10-\mathrm{C} 11-\mathrm{H} 11$ & 118.0 \\
\hline
\end{tabular}




$\begin{array}{llll}\mathrm{C} 5-\mathrm{C} 6-\mathrm{C} 1 & 117.1(2) & \mathrm{C} 7-\mathrm{O} 1-\mathrm{C} 4 & 116.05(18) \\ \mathrm{C} 5-\mathrm{C} 6-\mathrm{H} 6 & 121.4 & \mathrm{C} 7-\mathrm{N} 3-\mathrm{C} 11 & 117.8(2) \\ \mathrm{C} 1-\mathrm{C} 6-\mathrm{H} 6 & 121.4 & \mathrm{O} 4-\mathrm{N} 2-\mathrm{O} 5 & 124.2(2) \\ \mathrm{C} 1-\mathrm{C} 2-\mathrm{C} 3 & 118.2(3) & \mathrm{O} 4-\mathrm{N} 2-\mathrm{C} 8 & 118.75(19) \\ \mathrm{C} 1-\mathrm{C} 2-\mathrm{H} 2 & 120.9 & \mathrm{O} 5-\mathrm{N} 2-\mathrm{C} 8 & 117.0(2) \\ \mathrm{C} 3-\mathrm{C} 2-\mathrm{H} 2 & 120.9 & \mathrm{O} 2-\mathrm{N} 1-\mathrm{O} 3 & 123.3(3) \\ \mathrm{N} 3-\mathrm{C} 7-\mathrm{O} 1 & 118.66(19) & \mathrm{O} 2-\mathrm{N} 1-\mathrm{C} 1 & 118.2(3) \\ \mathrm{N} 3-\mathrm{C} 7-\mathrm{C} 8 & 122.1(2) & \mathrm{O} 3-\mathrm{N} 1-\mathrm{C} 1 & 118.4(3) \\ \mathrm{O} 1-\mathrm{C} 7-\mathrm{C} 8 & 119.2(2) & & \end{array}$

